Original Article Journal of Epilepsy Research pISSN 2233-6249 / eISSN 2233-6257

Received August 3, 2016

Accepted November 14, 2017

Corresponding author: Kishore K. Deepak Department of Physiology, All India Institute of Medical Sciences, Ansari Nagar, New Delhi110608, India

Tel. +91-011-2659 3583

Fax. $+91-011-26588663$, +91-011-2658 8789

E-mail; kkdeepak@gmail.com

\section{Comparison of Autonomic Function before and after Surgical Intervention in Patients with Temporal Lobe Epilepsy}

\author{
Navita Choudhary', Kishore K. Deepak ${ }^{1}$, Poodipedi S. Chandra ${ }^{2}$, Shalini Bhatia ${ }^{3}$, Rajesh Sagar ${ }^{4}$, \\ Ashok K. Jaryal ${ }^{1}$, Ravindra M. Pandey ${ }^{5}$, Manjari Tripathi ${ }^{6}$ \\ Departments of ${ }^{1}$ Physiology, ${ }^{2}$ Neurosurgery, ${ }^{4}$ Psychiatry, ${ }^{5}$ Biostatistics, ${ }^{6}$ Neurology, All India Institute of Medical \\ Sciences, New Delhi, India; ${ }^{3}$ Department of Research Support, A.T.Still University, Kirksville, MO, USA
}

Background and Purpose: Refractory temporal lobe epilepsy (TLE) is commonly associated with imbalances in cardiovascular (CV) parasympathetic and sympathetic functions, which are treated using TLE surgery. We investigated the effect of hemispheric lateralization of seizure foci on autonomic CV functions before and after TLE surgery.

Methods: The study was conducted on patients with left TLE (LTLE, $n=23$ ) and right TLE (RTLE, $n=30$ ) undergoing unilateral TLE surgery. To assess the autonomic CV functions, changes in the heart rate $(\triangle \mathrm{HR})$ and blood pressure (BP) were measured using a standardized battery of autonomic reactivity tests before surgery and at 3 and 6 months after surgery.

Results: Before surgery, $\triangle \mathrm{HR}$ and the expiration to inspiration ratio (E:I) during the deep breathing test were higher in the LTLE group than in the RTLE group (both $p<0.001$ ), but both outcomes were comparable between the groups at 3 and 6 months. $\Delta \mathrm{HR}$ decreased at 3 and 6 months ( $p<0.001$ and 0.01 , respectively) compared with preoperative values. The E:I at 3 months in the LTLE group was lower $(p=0.04)$ than the preoperative values. Decrease in systolic BP during the head-up tilt test was greater in the LTLE group than in the RTLE group $(p=0.002)$ before surgery. The maximum increase in diastolic BP during the cold pressor test was lower in the RTLE group at 6 months than that before surgery $(p=0.001)$ and in the LTLE group $(p=0.002)$.

Conclusions: We found that hemispheric lateralization of seizure foci in the temporal lobe had a differential effect on autonomic $\mathrm{CV}$ functions before surgery. Before surgery, parasympathetic reactivity was higher in the LTLE group, and sympathetic reactivity was higher in the RTLE group. After surgery, autonomic CV functions were comparable between the groups, suggesting that TLE surgery stabilizes autonomic CV functions.

(2017;7:89-98)

Key words: Epilepsy, temporal lobe, Hemispheric lateralization, Temporal lobe epilepsy surgery, Autonomic reactivity

\section{Introduction}

Temporal lobe epilepsy (TLE) may modify the functions of respiratory, gastrointestinal/abdominal, and cardiovascular (CV) autonomic regulation during the ictal and interictal period of seizures. ${ }^{1-5}$ During the interictal period, studies have reported an impairment of parasympathetic $^{6-9}$ and sympathetic ${ }^{7-11}$ CV functions. Studies have also shown that even a minimal epileptogenic discharge markedly disorganizes the autonomic nervous system, and such alterations in autonomic neural discharge are associated with arrhythmogenesis and may contribute to sudden death in patients with epilepsy. ${ }^{12,13}$

Previous studies have shown that the hemispheric center of autonomic functions differentially modulates autonomic CV function. During stressful tasks, healthy participants had increased blood flow in the right insula, which was associated with sympathetic activation. During non- tasks, left insular activation was observed, accompanied by decreases in blood pressure (BP) and heart rate (HR). ${ }^{14}$ Stimulation of the right insular cortex in patients with epilepsy revealed that the right hemisphere predominantly controlled sympathetic activity. ${ }^{15}$ Right hemispheric sympathetic control was also observed in studies 
where hemispheric inactivation was achieved using intracarotid amobarbital sodium. ${ }^{16-18}$ However, in many disease conditions, such as hemispheric stroke and lesion, the right hemisphere modulates parasympathetic functions. ${ }^{19-21}$

Hemispheric centers, particularly structures adjacent to the temporal lobe, play an important role in the regulation of autonomic CV functions. The adjacent interconnected structures of the temporal lobe, namely the insular cortex, amygdala, cingulate gyrus, and hippocampus, are the centers of autonomic CV modulation. ${ }^{22,23}$ Therefore, any destructive lesion such as an infarct, surgical resection, or stimulation such as epileptogenic activity in the temporal lobe may alter the autonomic modulation.

Therefore, we hypothesized that autonomic CV responses would be differentially affected in patients with left or right TLE before and after surgical resection of the seizure onset zone. Consequently, we investigated the effect of hemispheric lateralization of seizure foci on autonomic CV functions before and after TLE surgery. The effect of lateralization was assessed using a standardized battery of autonomic reactivity tests. Our study may contribute to a better understanding of the hemispheric modulation of autonomic CV function in patients with TLE before and after surgery.

\section{Methods}

\section{Patients}

The current prospective and observational cohort study was conducted in the Autonomic Function Lab at the All India Institute of Medical Sciences. Patients were referred from an intractable epilepsy clinic from January 2005 to September 2008 after the localization and lateralization of seizure foci. Only patients with refractory left TLE (LTLE) and right TLE (RTLE) who underwent surgery, were recruited. The study was approved by the local institutional ethics committee, and informed consent was obtained from each patient before testing. Patients with comorbidities that would affect autonomic functions, such as diabetes mellitus, cardiopulmonary disease, renal failure, and psychiatric illness, were excluded from the study. Chronic alcoholics and smokers were also excluded from the study.

The localization and lateralization of the seizure foci was assessed in the Department of Neurology by documenting at least 3 habitual seizures using video electroencephalography (VEEG). All patients had unilateral temporal lobe pathology with a same-sided seizure onset zone and underwent epilepsy surgery after routine pre-surgical evaluation. The pre-surgical evaluation included interictal electro- encephalography (EEG) (performed in triplicate), VEEG, ictally subtracted single-photon emission tomography, positron emission tomography, and magnetic resonance imaging (MRI, epilepsy protocol at 1.5 T).$^{24}$ At least 2 noninvasive evaluations, such as VEEG and MRI, had to be concordant before surgery. Patient eligibility for TLE surgery was decided after a discussion by a team of neurologists, neurosurgeons, neurophysiologists, neuroradiologists, and a neuropsychologist. Furthermore, patients underwent neuropsychological and psychiatric evaluations, visual field testing, and neurological diagnostic tests. Based on the pre-surgical evaluations and concordance for lateralization and localization, patients were divided into 2 groups: LTLE and RTLE groups. The following demographic information was collected: sex, age, type of seizures, duration of epilepsy, seizure frequency, Engel class of seizures, antiepileptic drugs (AED) taken, mean number of AEDs, EEG ictal onsets, etiology, and area resected during epilepsy surgery.

\section{Study procedures}

All autonomic function tests were conducted in an air-conditioned laboratory environment $\left(22-26^{\circ} \mathrm{C}\right)$ between $2 \mathrm{pm}$ and $4 \mathrm{pm}$ and 2 hours after a light meal. Autonomic function tests were conducted 3 times: before surgery and at 3 and 6 months after surgery. Autonomic function tests before surgery were conducted at least 12 hours after the last seizure and prior to hospitalization for surgery to avoid anxiety due to hospitalization. Patients received their regular AEDs on the testing days, and their drug regimen was not changed after surgery. All patients underwent a standard test battery for the assessment of autonomic CV reactivity as described previously. 25,26 The HR responses during the deep breathing test, Valsalva maneuver, and head-up tilt test were used as markers of parasympathetic reactivity, and BP responses to the hand grip test, cold pressor test, and head-up tilt test were used as markers of sympathetic reactivity.

\section{Resting measurements}

The resting $B P$, resting $H R$, and resting respiratory rate (RR) were measured after the patient rested in the supine position for 15 minutes. The BP was measured using a mercury sphygmomanometer, and $H R$ was measured using electrocardiography (ECG). The RR was measured using a chest stethograph (Model Vt. 159; INCO, Chandigarh, India). Both HR and RR were recorded continuously during autonomic reactivity tests using a standard polyrite machine (Recorders and Medicare, Chandigarh, India). 


\section{Parasympathetic reactivity tests}

For assessing parasympathetic function, changes in $\mathrm{HR}(\Delta \mathrm{HR})$ and the expiration to inspiration (E:I) ratio during the deep breathing test ( 6 breaths/minute with equal cycles of inspiratory/expiratory phase, each for 5 seconds) were used. The $\Delta H R$ was calculated as the difference between the maximum and minimum HR, and the E:I ratio was calculated by dividing the average of the longest RR interval by the smallest RR interval during a 6-breath/minute interval. For the Valsalva maneuver, the Valsalva ratio (VR) was calculated as the ratio of the longest RR interval to the shortest RR interval after and during blowing into a mercury manometer at a pressure of up to $40 \mathrm{mmHg}$ for 15 seconds. ${ }^{25,26}$ During head-up tilt test, the patients were tilted from horizontal to $70^{\circ}$ over 15 seconds and kept tilted for 5 minutes. The 30:15 ratio was calculated as the ratio of the longest $R R$ interval (around the 30th beat) to the shortest RR interval (around the 15th beat) on the ECG tracing obtained using the polyrite machine immediately after the $70^{\circ}$ tilt in the head-up tilt test.

\section{Sympathetic reactivity tests}

For assessing sympathetic function, a hand grip test was performed by using a hand grip dynamometer (Jetter and Scheerer, Tuttlingen, Germany) at 30\% of the patient's maximum voluntary contraction for up to 4 minutes, and BP was measured on the contra-lateral arm. ${ }^{25,26} \mathrm{~A}$ maximum increase in diastolic $\mathrm{BP}(\triangle \mathrm{DBP})$ from baseline was noted. The cold pressor test, in which the patient immersed his/her hand in cold water $\left(10^{\circ} \mathrm{C}\right)$ for 1 minute, was also assessed by noting $\triangle \mathrm{DBP}$ from baseline. For assessment using the head-up tilt test, a maximum fall in systolic BP from baseline was noted for up to 5 minutes after tilting using the intervals of $0.5 \mathrm{mi}$ nute, 1 minute, 2.5 minutes, and 5 minutes. ${ }^{25,26}$

Using a modified Engel scale, the surgical outcome was assessed at 6 months and 12 months after surgery. ${ }^{27}$ Seizure details were documented when patients visited for autonomic function testing at 6 months and over telephonic communication at 12 months. For the current study, class I patients on the Engle scale were seizure-free or had aura only, class II patients had rare seizures (> 95\% reduction), class III patients had 80-94\% reduction in seizures, and class IV patients had no worthwhile improvement $(<80 \%$ reduction in seizure activity).

\section{Statistical analysis}

Continuous variables were summarized as means \pm standard errors, and categorical data were summarized as frequencies. A 2-sam- ple $t$-test was used to compare the mean age, duration of epilepsy, seizure frequency, and number of AEDs given between the LTLE and RTLE groups. The categorical variables of Engel class and number of male patients in the 2 groups were analyzed using the $\chi^{2}$ test. Normality of the autonomic variables was verified using the Kolmogorov-Smirnoff test. To evaluate differences in the groups at the 3 time points (before surgery and at 3 and 6 months after surgery), random effects models (group $\times$ time interaction for all normally distributed variables) were built using the time of the measurement as a repeated factor. This analysis was performed for resting autonomic variables (systolic BP, diastolic BP, $H R$, RR), parasympathetic reactivity test variables ( $\triangle \mathrm{HR}$ during deep breathing test, E:I ratio during deep breathing test, VR during Valsalva maneuver, 30:15 during head-up tilt test), and sympathetic reactivity test variables ( $\triangle \mathrm{DBP}$ during hand grip test and cold pressor test). Most of the patients in our study had mesial temporal sclerosis (MTS). Because uniformity of condition may provide more information, we performed a subgroup analysis of left MTS (LMTS) and right MTS (RMTS) patients only. Therefore, a similar analysis was performed for all the above variables to evaluate differences in the LMTS and RMTS subgroups at the 3 time points. To evaluate the difference in the fall of systolic BP during the head-up tilt test between the LTLE and RTLE groups separately at the 3 time points, a Wilcoxon rank sum test was performed. A within-group analysis was performed to compare the fall in systolic BP at the 3 time points separately in each group (LTLE and RTLE) using Friedman's test, and a similar protocol was followed for the LMTS and RMTS subgroups. Post-hoc analyses for within-group comparisons at various time points and between groups were performed using Scheffe's adjustment to type I error. A $p$ value of $<0.05$ was considered statistically significant. Data were analyzed utilizing SAS version 9.4 (SAS Institute Inc., Cary, NC, USA).

\section{Results}

Twenty-three LTLE patients (14 men, 9 women; age, $23.56 \pm$ 11.31 years) and 30 RTLE patients (18 men, 12 women; age, $22.4 \pm$ 12.29 years) completed the study. No differences were found in the mean age ( $p=0.53$ ) or in the sex distribution between the 2 groups $(p=0.90)$. The demographic characteristics of the patients are summarized in Table 1. No differences were found in the type of seizure, duration of epilepsy, seizure frequency, Engel class, and number of AEDs between the groups. Sixteen patients had LMTS, and 23 had RMTS. The etiology in the 2 groups and the type of TLE surgery per- 
Table 1. Summary of the clinical variables observed in study participants

\begin{tabular}{lccc}
\hline Clinical variable & LTLE group $(\mathrm{n}=23)$ & RTLE group $(\mathrm{n}=30)$ & $p$-value \\
\hline Type of seizure & 21 & 28 & NA \\
CPS & 2 & 2 & - \\
CPS + sGTC & $12.28 \pm 6.38$ & $10.68 \pm 6.34$ & - \\
Duration of epilepsy (years) & $6.83 \pm 7.51$ & $5.87 \pm 7.35$ & 0.37 \\
Seizure frequency (months) & & & 0.64 \\
Engel class & 21 & 27 & -36 \\
Class I & 2 & 3 & - \\
Class II & & & NA \\
AED taken & 3 & 6 & - \\
Levetiracetam & 9 & 13 & - \\
Valproate & 14 & 30 & - \\
Carbamazepine & 10 & 5 & - \\
Clobazam & 4 & 6 & - \\
Phenytoin & 6 & 8 & - \\
Lamotrigine & 1 & 3 & - \\
Phenobarbital & 3 & 3 & - \\
Topiramate & $2.26 \pm 0.45$ & $2.50 \pm 0.51$ & NA \\
Number of AEDs & & & - \\
EEG ictal onsets & 20 & 25 & - \\
Anterior temporal & 3 & 5 & \\
Anterior and mid temporal & & & \\
\hline
\end{tabular}

Values are presented as frequencies or means \pm standard errors. Categorical data were analyzed using a $\chi^{2}$ test, and continuous data were analyzed using a 2-sample $t$-test.

LTLE, left temporal lobe epilepsy; RTLE, right temporal lobe epilepsy; NA, not applicable; CPS, complex partial seizure; sGTC, secondary generalized tonic clonic seizure; AED, antiepileptic drug; EEG, electroencephalography.

*Patients were taking multiple drugs at one time.

Table 2. Etiology and type of surgery performed

\begin{tabular}{llcc}
\hline Area resected & Etiology & LTLE $(n=23)$ & RTLE $(n=30)$ \\
\hline Anterior mesial temporal lobectomy & Unilateral mesial temporal sclerosisatrophy limited to the hippocampus & 16 & 23 \\
Temporal lobectomy & $\begin{array}{l}\text { Post-traumatic temporal lobe gliosis, post infarct encephalomalacia } \\
\text { involving the temporal lobe }\end{array}$ & 5 & 5 \\
Lesionectomy & Dysembryoplastic neuroepithelial tumor, cavernoma, ganglioglioma & 2 & 2 \\
\hline
\end{tabular}

LTLE, left temporal lobe epilepsy; RTLE, right temporal lobe epilepsy.

formed is presented in Table 2.

No significant group $\times$ time interaction was found for resting autonomic CV variables: systolic $\mathrm{BP}(p=0.82)$, diastolic $\mathrm{BP}(p=0.37)$, $\operatorname{HR}(p=0.09)$, and RR $(p=0.97)$. Summary data for the autonomic CV function parameters are presented in Table 3. When comparing the LMTS and RMTS subgroups, no significant group $\times$ time interaction was found: systolic $\mathrm{BP}(p=0.87)$, diastolic $\mathrm{BP}(p=0.92)$, HR $(p=0.23)$, and RR $(p=0.84)$.

For the variables of parasympathetic reactivity, a significant group $\times$ time interaction was found during the deep breathing test for $\Delta \mathrm{HR}$ $p<0.001)$ and the E:I ratio $(p=0.03)$. Before surgery, $\Delta \mathrm{HR}$ and $\mathrm{E}: \mathrm{I}$ (both $p<0.001$ ) were higher in the LTLE group than in the RTLE group (Table 4). At 3 and 6 months after surgery, $\Delta H R$ during the deep breathing test for the LTLE group significantly decreased compared to the values before surgery (both $p<0.01$ ). The E:I ratio for the LTLE group significantly decreased after 3 months compared to the ratio before surgery $(p=0.04)$. For the LMTS and RMTS subgroup analysis, a significant group $\times$ time interaction was found during the deep breathing test for $\Delta \mathrm{HR}(p<0.001)$ and $\mathrm{E}: \mathrm{I}(p=0.046)$. Before surgery, $\Delta$ HR and E:I (both $p<0.001$ ) were higher in the LMTS subgroup than in the RMTS subgroup. After 3 months, $\Delta H R$ for the LMTS group had significantly decreased from that before surgery $(p=$ $0.02)$. No significant group $\times$ time interaction was found for the VR during the Valsalva maneuver $(p=0.09)$ or 30:15 ratio during the head-up tilt test $(p=0.16)$.

For the variables of sympathetic reactivity, no significant group $\times$ time interaction was found for $\triangle \mathrm{DBP}$ during the hand grip test for the LTLE and RTLE groups $(p=0.46)$ or the LMTS and RMTS subgroups ( $p=0.71)$. A significant group $\times$ time interaction was found during the cold pressor test for the LTLE and RTLE groups $(p=0.005)$. 
Table 3. Resting autonomic variables before surgery and at 3 and 6 months after surgery

\begin{tabular}{|c|c|c|c|c|c|}
\hline Variable & $\begin{array}{c}\text { Visit } \\
\text { (months) }\end{array}$ & $\begin{array}{c}\text { LTLE } \\
(n=23)\end{array}$ & $\begin{array}{c}\text { RTLE } \\
(n=30)\end{array}$ & $\begin{array}{c}\text { LMTS } \\
(n=16)\end{array}$ & $\begin{array}{c}\text { RMTS } \\
(n=23)\end{array}$ \\
\hline \multicolumn{6}{|c|}{ Systolic BP (mmHg) } \\
\hline & 0 & $114.45 \pm 3.21$ & $113.19 \pm 2.48$ & $112.98 \pm 4.23$ & $114.70 \pm 3.14$ \\
\hline & 3 & $117.37 \pm 4.33$ & $115.60 \pm 3.27$ & $118.67 \pm 5.75$ & $117.54 \pm 4.10$ \\
\hline & 6 & $109.90 \pm 3.81$ & $111.98 \pm 3.04$ & $108.72 \pm 3.72$ & $111.66 \pm 3.01$ \\
\hline \multicolumn{6}{|c|}{ Diastolic BP (mmHg) } \\
\hline & 0 & $73.84 \pm 3.13$ & $70.52 \pm 2.41$ & $72.26 \pm 4.13$ & $72.00 \pm 3.05$ \\
\hline & 3 & $74.58 \pm 3.46$ & $74.29 \pm 2.60$ & $73.98 \pm 4.55$ & $75.36 \pm 3.22$ \\
\hline & 6 & $63.38 \pm 3.63$ & $68.25 \pm 2.81$ & $64.90 \pm 4.60$ & $67.84 \pm 3.56$ \\
\hline \multicolumn{6}{|l|}{ HR (bpm) } \\
\hline & 0 & $81.21 \pm 2.55$ & $80.04 \pm 1.96$ & $79.11 \pm 2.52$ & $76.45 \pm 1.88$ \\
\hline & 3 & $79.29 \pm 4.43$ & $85.22 \pm 3.32$ & $84.91 \pm 5.22$ & $86.34 \pm 3.70$ \\
\hline & 6 & $73.93 \pm 2.95$ & $82.56 \pm 2.29$ & $76.84 \pm 3.26$ & $81.45 \pm 2.53$ \\
\hline \multicolumn{6}{|l|}{$\mathrm{RR}$ (cpm) } \\
\hline & 0 & $19.44 \pm 1.11$ & $17.85 \pm 0.86$ & $20.46 \pm 1.37$ & $17.50 \pm 1.02$ \\
\hline & 3 & $17.80 \pm 0.81$ & $16.57 \pm 0.61$ & $18.64 \pm 0.78$ & $16.69 \pm 0.55$ \\
\hline & 6 & $18.38 \pm 0.95$ & $17.26 \pm 0.73$ & $18.94 \pm 0.95$ & $16.73 \pm 0.74$ \\
\hline
\end{tabular}

Values are presented as mean \pm standard errors, which were calculated from random effects models with time as a repeated factor using the GLIMMIX procedure in SAS. Visit 0 was before surgery; visits 3 and 6 were at 3 and 6 months after the surgery, respectively. LTLE, left temporal lobe epilepsy; RTLE, right temporal lobe epilepsy; LMTS, left mesial temporal lobe sclerosis; RMTS, right mesial temporal lobe sclerosis; BP, blood pressure; HR, heart rate; bpm, beats per minute; RR, respiratory rate; cpm, cycles per minute.

Table 4. Parasympathetic reactivity tests before surgery and at 3 and 6 months after surgery

\begin{tabular}{|c|c|c|c|c|c|c|c|}
\hline Variable & $\begin{array}{c}\text { Visit } \\
\text { (months) }\end{array}$ & $\begin{array}{c}\text { LTLE } \\
(n=23)\end{array}$ & $\begin{array}{c}\text { RTLE } \\
(n=30)\end{array}$ & $p$-value & $\begin{array}{c}\text { LMTS } \\
(n=16)\end{array}$ & $\begin{array}{c}\text { RMTS } \\
(n=23)\end{array}$ & $p$-value ${ }^{\dagger}$ \\
\hline \multicolumn{8}{|c|}{$\Delta \mathrm{HR}$ during deep breathing test } \\
\hline & 0 & $32.33 \pm 1.94$ & $18.86 \pm 1.55$ & $<0.001$ & $32.70 \pm 2.58$ & $18.00 \pm 1.94$ & $<0.001$ \\
\hline & 3 & $22.40 \pm 2.01$ & $21.24 \pm 1.46$ & 0.64 & $23.38 \pm 2.60$ & $22.34 \pm 1.81$ & 0.74 \\
\hline & 6 & $23.08 \pm 2.95$ & $21.17 \pm 2.25$ & 0.61 & $24.38 \pm 3.76$ & $20.95 \pm 2.82$ & 0.47 \\
\hline \multicolumn{8}{|l|}{$p$-value ${ }^{\ddagger}$} \\
\hline & 0 vs. 3 & $<0.001$ & 0.40 & & 0.02 & 0.13 & \\
\hline & 0 vs. 6 & 0.01 & 0.59 & & 0.11 & 0.57 & \\
\hline \multicolumn{8}{|c|}{ E:I ratio during deep breathing test } \\
\hline & 0 & $1.44 \pm 0.03$ & $1.28 \pm 0.02$ & $<0.001$ & $1.48 \pm 0.03$ & $1.30 \pm 0.03$ & $<0.001$ \\
\hline & 3 & $1.29 \pm 0.05$ & $1.32 \pm 0.04$ & 0.75 & $1.32 \pm 0.06$ & $1.34 \pm 0.05$ & 0.81 \\
\hline & 6 & $1.30 \pm 0.05$ & $1.29 \pm 0.04$ & 0.85 & $1.31 \pm 0.06$ & $1.28 \pm 0.05$ & 0.79 \\
\hline \multicolumn{8}{|l|}{$p$-value } \\
\hline & 0 vs. 3 & 0.04 & 0.75 & & 0.07 & 0.76 & \\
\hline & 0 vs. 6 & 0.09 & 0.99 & & 0.08 & 0.96 & \\
\hline \multicolumn{8}{|c|}{ VR during Valsalva maneuver } \\
\hline & 0 & $1.98 \pm 0.13$ & $1.83 \pm 0.09$ & $N A^{\S}$ & $1.98 \pm 0.15$ & $1.89 \pm 0.12$ & $N A^{\S}$ \\
\hline & 3 & $1.74 \pm 0.14$ & $1.56 \pm 0.10$ & $N A^{\S}$ & $1.74 \pm 0.18$ & $1.56 \pm 0.13$ & $N A^{\S}$ \\
\hline & 6 & $1.78 \pm 0.12$ & $1.69 \pm 0.09$ & $N A^{\S}$ & $1.78 \pm 0.13$ & $1.77 \pm 0.10$ & $N A^{\S}$ \\
\hline \multicolumn{8}{|l|}{$p$-value } \\
\hline & 0 vs. 3 & $N A^{\S}$ & $N A^{\S}$ & & $N A^{\S}$ & $N A^{\S}$ & \\
\hline \multicolumn{2}{|c|}{$30: 15$ ratio during head-up tilt test } & $N A^{\S}$ & $N A^{\S}$ & & $N A^{\S}$ & $N A^{\S}$ & \\
\hline & 0 & $1.12 \pm 0.02$ & $1.13 \pm 0.02$ & $N A^{\S}$ & $1.16 \pm 0.03$ & $1.13 \pm 0.02$ & $N A^{\S}$ \\
\hline & 3 & $1.10 \pm 0.04$ & $1.19 \pm 0.03$ & $N A^{\S}$ & $1.09 \pm 0.04$ & $1.16 \pm 0.03$ & $N A^{\S}$ \\
\hline & 6 & $1.14 \pm 0.03$ & $1.11 \pm 0.02$ & $N A^{\S}$ & $1.14 \pm 0.03$ & $1.11 \pm 0.03$ & $N A^{\S}$ \\
\hline \multicolumn{8}{|l|}{$p$-value } \\
\hline & 0 vs. 3 & $N A^{\S}$ & $N A^{\S}$ & & 0.52 & $N A^{\S}$ & \\
\hline & 0 vs. 6 & $N A^{\S}$ & $N A^{\S}$ & & 0.95 & $N A^{\S}$ & \\
\hline
\end{tabular}

All data except the $p$-values are presented as mean \pm standard errors. Visit 0 was before surgery; visits 3 and 6 were 3 and 6 months after the surgery, respectively.

LTLE, left temporal lobe epilepsy; RTLE, right temporal lobe epilepsy; LMTS, left mesial temporal lobe sclerosis; RMTS, right mesial temporal lobe sclerosis; $\triangle \mathrm{HR}$, change in heart rate; $\mathrm{E}: \mathrm{I}$ ratio, expiratory:inspiratory ratio during the deep breathing test; VR, Valsalva ratio; NA, not applicable; 30:15 ratio, ratio of the longest RR interval (around the 30th beat) to the shortest RR interval (around the 15th beat) on the ECG tracing after the $70^{\circ}$ tilt during the head-up tilt test.

*Comparison of LTLE vs. RTLE. p-values obtained from random effects model using the GLIMMIX procedure in SAS with time as a repeated factor; ${ }^{\dagger}$ Comparison of LMTS vs. RMTS. p-values obtained from random effects model using the GLIMMIX procedure in SAS with time as a repeated factor; "Scheffe's adjustment to $p$-value applied for multiple comparisons to control type I error rate; ${ }^{\S}$ Because the group $\times$ time interaction did not show a significant difference and additional testing was not necessary. 
94 Journal of Epilepsy Research Vol. 7, No. 2, 2017

Table 5. Sympathetic reactivity tests before surgery and at 3 and 6 months after surgery

\begin{tabular}{|c|c|c|c|c|c|c|c|}
\hline Variable & $\begin{array}{c}\text { Visit } \\
\text { (months) }\end{array}$ & $\begin{array}{c}\text { LTLE } \\
(n=23)\end{array}$ & $\begin{array}{c}\text { RTLE } \\
(n=30)\end{array}$ & $p$-value* & $\begin{array}{c}\text { LMTS } \\
(n=16)\end{array}$ & $\begin{array}{c}\text { RMTS } \\
(n=23)\end{array}$ & $p$-value \\
\hline \multicolumn{8}{|c|}{$\triangle \mathrm{DBP}$ during hand grip test } \\
\hline & 0 & $19.31 \pm 1.91$ & $19.26 \pm 1.52$ & $N A^{\ddagger}$ & $20.11 \pm 2.45$ & $19.58 \pm 1.86$ & $N A^{\ddagger}$ \\
\hline & 3 & $20.69 \pm 2.81$ & $19.00 \pm 2.17$ & $N A^{\ddagger}$ & $20.67 \pm 3.69$ & $18.70 \pm 2.69$ & $N A^{*}$ \\
\hline & 6 & $25.34 \pm 3.25$ & $19.59 \pm 2.47$ & $N A^{\ddagger}$ & $24.92 \pm 4.06$ & $19.59 \pm 3.08$ & $N A^{*}$ \\
\hline \multicolumn{8}{|l|}{$p$-value } \\
\hline & 0 vs. 3 & $N A^{\ddagger}$ & $N A^{\ddagger}$ & & $N A^{\ddagger}$ & $N A^{\ddagger}$ & \\
\hline & 0 vs. 6 & $N A^{\ddagger}$ & $N A^{\ddagger}$ & & $N A^{\ddagger}$ & $N A^{\ddagger}$ & \\
\hline \multicolumn{8}{|c|}{$\triangle \mathrm{DBP}$ during cold pressor test } \\
\hline & 0 & $16.12 \pm 2.18$ & $18.61 \pm 1.72$ & 0.38 & $16.93 \pm 2.80$ & $19.34 \pm 2.15$ & 0.50 \\
\hline & 3 & $15.00 \pm 3.58$ & $23.52 \pm 2.83$ & 0.07 & $13.62 \pm 4.68$ & $24.35 \pm 3.41$ & 0.07 \\
\hline & 6 & $19.38 \pm 2.67$ & $8.59 \pm 1.93$ & 0.002 & $22.37 \pm 2.96$ & $9.52 \pm 2.09$ & 0.002 \\
\hline \multicolumn{8}{|l|}{$p$-value ${ }^{\S}$} \\
\hline & 0 vs. 3 & 0.96 & 0.25 & & 0.80 & 0.37 & \\
\hline & 0 vs. 6 & 0.64 & 0.001 & & 0.45 & 0.02 & \\
\hline \multicolumn{8}{|c|}{$\begin{array}{l}\text { Fall in systolic BP during head-up } \\
\text { tilt test }\end{array}$} \\
\hline & 0 & $8.67 \pm 1.46$ & $2.67 \pm 1.19$ & 0.002 & $10.46 \pm 1.83^{\dagger}$ & $3.60 \pm 1.48$ & 0.008 \\
\hline & 3 & $12.98 \pm 2.93$ & $6.49 \pm 2.20$ & 0.15 & $15.69 \pm 3.67$ & $5.32 \pm 2.58$ & 0.06 \\
\hline & 6 & $8.89 \pm 2.51$ & $6.65 \pm 1.90$ & 0.22 & $5.80 \pm 3.07$ & $6.74 \pm 2.31$ & 0.61 \\
\hline \multicolumn{8}{|l|}{$p$-value ${ }^{\|}$} \\
\hline & 0 vs. 3 & $N S^{n}$ & $N S^{n}$ & & $N S^{n}$ & $N S^{n}$ & \\
\hline & 0 vs. 6 & $N S^{n}$ & $N S^{n}$ & & $N S^{n}$ & $N S^{n}$ & \\
\hline
\end{tabular}

All data except the $p$-values are presented as mean \pm standard errors. Visit 0 was before surgery; visits 3 and 6 were 3 and 6 months after the surgery, respectively.

LTLE, left temporal lobe epilepsy; RTLE, right temporal lobe epilepsy; LMTS, left mesial temporal lobe sclerosis; RMTS, right temporal lobe sclerosis; $\triangle \mathrm{DBP}$, maximum increase in diastolic blood pressure; NA, not applicable; BP, blood pressure; NS, not significant.

${ }^{*}$ Comparison of LTLE vs. RTLE. $p$-values obtained from random effects model using the GLIMMIX procedure in SAS with time as a repeated factor; ${ }^{\dagger}$ Comparison of LMTS vs. RMTS. p-values obtained from random effects model using the GLIMMIX procedure in SAS with time as a repeated factor; "Because the group $\times$ time interaction did not show a significant difference and additional testing was not necessary; $\S S$ cheffe's adjustment to $p$-value applied for multiple comparisons to control type I error rate; "Wilcoxon rank sum test to calculate $p$-values for fall in systolic BP during head-up tilt test to test for group differences at various time points; "Used to maintain homogeneity for all sympathetic variables in the table.

At 6 months, $\triangle \mathrm{DBP}$ during the cold pressor test was significantly lower $(p=0.002)$ in the RTLE group than in the LTLE group. The $\triangle \mathrm{DBP}$ was also significantly lower at 6 months than that before surgery in the RTLE group ( $p=0.001$; Table 5 ). When comparing the LMTS and RMTS subgroups, a significant group $\times$ time interaction was found for $\triangle \mathrm{DBP}$ during the cold pressor test $(p=0.009)$. At 6 months, $\triangle \mathrm{DBP}(p=0.002)$ was lower in the RMTS group than in the LMTS group and was significantly decreased from that before surgery $(p=0.02)$ in the RMTS group. Before surgery, the fall in systolic BP during head-up tilt test was higher in the LTLE group than in the RTLE group $(p=0.002)$. No within-group differences at any time point were found. In the subgroup analysis, differences were found between the LMTS and RMTS groups before surgery for the head-up tilt test $(p=0.008)$; the fall in systolic BP was lower in the RMTS subgroup than in the LMTS subgroup. However, differences were not significant 3 or 6 months after surgery. In the within-group analysis, no difference was found in the fall in systolic BP during the head-up tilt test between the LMTS $(p=0.27)$ and RMTS $(p=0.53)$ subgroups at any of the 3 time points.

Eight patients in the LTLE group and 14 patients in the RTLE group were unable to perform the hand grip test and cold pressor test according to the protocol. During the hand grip test with these patients, BP was measured 2-4 minutes after the patient pressed the hand grip dynamometer rather than at the full 4 minutes. During the cold pressor test, these patients were unable to keep their hand in cold water $\left(10^{\circ} \mathrm{C}\right)$ for 1 minute, and $\mathrm{BP}$ was thus measured approximately 25-35 seconds after the patient's hand was placed in the cold water.

\section{Discussion}

The current study showed that hemispheric sidedness of the seizure onset zone had a differential effect on autonomic CV functions in patients with TLE. We found that before surgery, patients having 
Seizure foci in the left temporal lobe had higher parasympathetic reactivity than that of patients with seizure foci in the right temporal lobe. After surgery, parasympathetic reactivity was lower in the LTLE group and LMTS subgroup than that before surgery. Moreover, at 3 and 6 months after surgery, the LTLE and RTLE groups and the LMTS and RMTS subgroups had comparable parasympathetic reactivity. Before surgery, patients with seizure foci in the right temporal lobe had a higher sympathetic reactivity than patients with seizure foci in the left temporal lobe. On the basis of these findings, we suggest that LTLE increases the parasympathetic response to HR, and TLE surgery reduces this heightened response. Therefore, LTLE surgery may help in stabilizing parasympathetic functions. We also suggest that epilepsy in the right temporal lobe increases the sympathetic response to BP. Thus, RTLE surgery may reduce this heightened response, although additional studies are required to substantiate this finding.

In general, the $\Delta H R$ and the E:I ratio are robust markers of parasympathetic reactivity. ${ }^{28,29}$ In the current study, higher values of these variables in the LTLE group and LMTS subgroup indicated a higher parasympathetic response to HR than that of the RTLE group and RMTS subgroup, respectively. However, these variables decreased after surgery and became comparable among the groups. These findings suggested that patients with LTLE have heightened parasympathetic control of HR, which may cause bradycardia, hypotension, and asystole during the ictal period of seizures, as reported in various studies. ${ }^{30,31}$ Furthermore, our results suggested that TLE surgery in the LTLE group and LMTS subgroup may reduce these cardiac risks and improve autonomic function in patients with TLE.

A fall in systolic BP during the head-up tilt test is a marker of sympathetic reactivity. A lower fall of systolic BP suggests higher sympathetic activation during the head-up tilt test. ${ }^{32,33}$ In the current study, the smaller fall in systolic BP during the head-up tilt test in the RTLE group suggested higher sympathetic function than that in the LTLE group. However, both groups showed similar sympathetic reactivity after surgery. Furthermore, the fall in systolic BP in the RTLE group showed a tendency to increase after surgery. This finding suggests that RTLE surgery mitigates the heightened sympathetic function. Similarly, $\triangle \mathrm{DBP}$ during the cold pressor test is a marker of sympathetic function, wherein a higher $\triangle \mathrm{DBP}$ indicates increased sympathetic function. ${ }^{34}$ In the current study, the LTLE and RTLE groups had comparable $\triangle \mathrm{DBP}$ during the cold pressor test before surgery, but $\triangle \mathrm{DBP}$ significantly decreased at 6 months in the RTLE group and RMTS subgroup compared to that before surgery. Additionally, $\triangle \mathrm{DBP}$ was significantly lower at 6 months after surgery in the RTLE groups than in the LTLE group and LMTS subgroup. This finding also indicated that sympathetic reactivity was reduced after RTLE surgery.

Consistent with our present findings, a previous study ${ }^{35}$ examining patients with hippocampal sclerosis associated with TLE found that before surgery, patients with hippocampal sclerosis and LTLE had elevated parasympathetic response, while those with RTLE had elevated sympathetic response, which mediated excessive bradycardia and tachycardia counter-regulation. To understand the mechanism of autonomic modulation in patients with LTLE and RTLE, we included 2 time points after surgery and found that surgical removal of the affected lesion or area influenced autonomic CV modulation differentially. The LTLE group and LMTS subgroup showed a significant decrease in parasympathetic response after surgery, while the RTLE group and RMTS subgroup tended to show a decrease in heightened sympathetic responses, which may consequently decrease the risk of cardiac emergencies in TLE.

Changes in autonomic CV modulation at different time points after surgery have been reported in previous studies. ${ }^{36-40}$ One study ${ }^{37}$ reported sympathetic over-activity 1 month after a temporal lobectomy, and other studies ${ }^{36,40}$ demonstrated a decrease in sympathetic CV modulation in patients with TLE at 3-4 months after surgery. Previous findings have also suggested that patients with good outcomes 12 months after surgery exhibited a decrease in sympathetic $\mathrm{CV}$ modulation at 6 months, while patients with poor outcomes showed no change. ${ }^{38,39}$ In the current study, all patients were seizure-free at 12 months after surgery. We found a change in parasympathetic modulation as early as 3 months after surgery, and a change in sympathetic modulation was also observed at 6 months after surgery. This variation in the changes in autonomic function at different time points may have been affected by various factors like seizure severity, ${ }^{5}$ epileptic drugs, ${ }^{7,11}$ type of surgery, the actual surgery, number of patients, and the methods used to assess the autonomic functions. However, other unknown and uncontrolled factors may have also influenced these results. Future studies should be conducted with a longer follow-up period to understand the effect of time on this variation.

In the current study, only $\Delta H R$ and E:I ratio during the deep breathing test showed significant interaction for the parasympathetic response. The VR assessed using the Valsalva maneuver and 30:15 ratio during the head-up tilt test, which are markers of parasympathetic function, showed no significant interaction of group and time. Because the VR indicates the interaction of sympathetic, para- 
sympathetic, and baroreflex response, ${ }^{41}$ this versatility may explain our results for this variable. We suggest that future studies use different methods for assessing autonomic functions, such as HR variability and baroreflex sensitivity.

Our findings on the sympathetic response were based on a single autonomic function test, namely, the head-up tilt test. The other autonomic function tests for sympathetic reactivity we used were not successful because patients had difficulty performing the hand grip test, which requires some effort, and the cold pressor test, which requires the ability to tolerate cold. These challenges during the hand grip and cold pressor tests limited the value of our results with reference to non-baroreflex-based autonomic responses. Therefore, future studies should consider using a robust sympathetic activity marker, such as muscle sympathetic nerve activity, to assess sympathetic function in conjunction with simultaneous EEG recordings.

Previous studies have suggested that AEDs like carbamazepine may suppress parasympathetic and sympathetic functions in patients with epilepsy. ${ }^{7,11}$ However, another study found that carbamazepine had no association with decreased autonomic functions in patients with epilepsy. ${ }^{42}$ In the current study, 14 patients in the LTLE group and 30 in the RTLE group were administered carbamazepine, which may have influenced our results.

Because a majority of the patients with epilepsy in the current study had MTS, we performed a subgroup analysis (LMTS and RMTS) to determine if these subgroups had distinct results from the main TLE groups (LTLE and RTLE) that included all types of insults in the temporal lobe. We found that the parasympathetic and sympathetic modulations were similar in both groups (TLE groups and MTS subgroups) before surgery. After surgery, there were changes in the significance within the MTS subgroups although the mean values were still comparable with those of the main group (TLE). We believe this change in significance in the MTS subgroups was due to the fewer number of patients in those subgroups, which contributed to the high variation in the mean values. In the temporal lobe, all parts of the structure are interconnected and associated with the modulation of the autonomic nervous system; ${ }^{3,22}$ any insult in one region may affect the function of other neighboring structures. This interconnectedness could be one of the reasons for the observed comparable results between the TLE groups and MTS subgroups. A larger study with a larger sample size is required to confirm if these changes are maintained in the MTS subgroups.

The epileptogenic discharges that arise from the temporal lobe during the ictal or interictal period of seizures may interfere with au- tonomic CV modulation because the interconnected structures of the temporal lobe, such as the insular cortex, amygdala, cingulate gyrus, and hippocampus, and their pathways play an important role in the regulation of autonomic CV modulation. ${ }^{3,22}$ Similarly, surgical removal of the seizure foci may alter autonomic CV functions. Studies demonstrated an increase in cerebral glucose metabolism after TLE surgery in areas of the autonomic nervous system. ${ }^{43,44}$ However, the mechanism of epileptogenic activity in TLE before and after surgery is unclear. The pathophysiology and clinical manifestations associated with TLE are very complex and may represent the combined effect of hyper-excitation observed with seizures and hypo-function from loss of neuronal tissue in the affected brain regions.

The observed heightened parasympathetic and sympathetic responses before epilepsy surgery in the current study may be attributed to the presence of subclinical seizures during autonomic function tests. It has been reported that even minimal epileptogenic activity can alter cardiac neural discharge and arrhythmia. ${ }^{12}$ Therefore, any subclinical epileptogenic activity may alter the functions of different areas of the brain, such as the cerebral cortex or amygdala, and may result in changes in the cardiac autonomic function. We could not use EEG in the present study because it was not feasible during testing; thus, we conducted the tests at least 12 hours after the last seizure (according to the patient's verbal report) to obtain information about subclinical epileptogenic activities.

In summary, the comparatively higher parasympathetic response in the LTLE group and the higher sympathetic response in the RTLE group suggested lateralization of the hemispheric center of autonomic CV controls in patients with epilepsy. Furthermore, TLE surgery seemed to stabilize autonomic $\mathrm{CV}$ regulation by reducing the parasympathetic CV responses in the LTLE group and LMTS subgroup; the results also indicated that surgery may reduce sympathetic $\mathrm{CV}$ responses in the RTLE group and RMTS subgroup. Given these results, we believe that lower parasympathetic outflow in LTLE and sympathetic outflow in RTLE should be accompanied by a reduced risk of CV emergencies after surgery in patients with TLE.

\section{Acknowledgement}

The author was funded by a doctoral fellowship from the University Grant Commission (UGC), Government of India, to conduct this work. 


\section{Conflict of Interest}

All the authors declare no conflict of interests.

\section{References}

1. Van Buren JM, Ajmone-Marson C. A correlation of autonomic and EEG components in temporal lobe epilepsy. Arch Neurol 1960;3:683-703.

2. Freeman R, Schachter SC. Autonomic epilepsy. Semin Neurol 1995;15:158-66.

3. Benarroch EE. Telencephalic disorders. In: Benarroch EE, ed. Central Autonomic Network: Functional organization and clinical correlations. Armonk: Futura Publishing Company, 1997;539-559.

4. Spyer KM. Central nervous control of cardiovascular system. In: Mathias CJ, Bannister R, eds. Autonomic Failure: A text book of clinical disorders of the autonomic nervous system, 4th ed. Oxford: Oxford University Press, 1999;45-55.

5. Mukherjee S, Tripathi M, Chandra PS, et al. Cardiovascular autonomic functions in well-controlled and intractable partial epilepsies. Epilepsy Res 2009;85:261-9.

6 Drake ME Jr, Andrews JM, Castleberry CM. Electrophysiologic assessment of autonomic function in epilepsy. Seizure 1998;7:91-6.

7. Isojärvi Jl, Ansakorpi $H$, Suominen K, Tolonen U, Repo M, Myllylä W. Interictal cardiovascular autonomic responses in patients with epilepsy. Epilepsia 1998:39:420-6.

8. Novak W, Reeves LA, Novak P, Low AP, Sharbrough WF. Time-frequency mapping of R-R interval during complex partial seizures of temporal lobe origin. J Auton Nerv Syst 1999;77:195-202.

9. Ansakorpi H, Korpelainen JT, Suominen K, Tolonen U, Myllylä W, Isojärvi II. Interictal cardiovascular autonomic responses in patients with temporal lobe Epilepsy. Epilepsia 2000;41:42-7.

10. Massetani R, Strata G, Galli R, et al. Alteration of cardiac function in patients with temporal lobe epilepsy: different role of EEG-ECG monitoring and spectral analysis of RR variability. Epilepsia 1997;38:363-9.

11. Tomson $T$, Ericson M, Ihrman C, Lindblad LE. Heart rate variability in patients with epilepsy. Epilepsy Res 1998;30:77-83.

12. Lathers CM, Schraeder PL. Autonomic dysfunction in epilepsy: characterization of autonomic cardiac neural discharge associated with pentylenetetrazol-induced epileptogenic activity. Epilepsia 1982;23:633-47.

13. Lathers CM, Schraeder PL, Weiner FL. Synchronization of cardiac autonomic neural discharge with epileptogenic activity: the lockstep phenomenon. Electroencephalogr Clin Neurophysio/ 1987;67:247-59.

14. Critchley HD, Corfield DR, Chandler MP, Mathias CJ, Dolan RJ. Cerebral correlates of autonomic cardiovascular arousal: a functional neuroimaging investigation in humans. J Physio/ 2000;523 Pt 1:259-70.

15. Oppenheimer SM, Gelb A, Girvin JP, Hachinski VC. Cardiovascular effects of human insular cortex stimulation. Neurology 1992;42:1727-32.

16. Zamrini EY, Meador KJ, Loring DW, et al. Unilateral cerebral inactivation produces differential left/right heart rate responses.
Neurology 1990;40:1408-11.

17. Yoon BW, Morillo CA, Cechetto DF, Hachinski V. Cerebral hemispheric lateralization in cardiac autonomic control. Arch Neurol 1997;54:741-4.

18. Hilz MJ, Dütsch $M$, Perrine K, Nelson PK, Rauhut U, Devinsky 0. Hemispheric influence on autonomic modulation and baroreflex sensitivity. Ann Neurol 2001;49:575-84.

19. Barron SA, Rogovski Z, Hemli J. Autonomic consequences of cerebral hemisphere infarction. Stroke 1994;25:113-6.

20. Naver HK, Blomstrand C, Wallin BG. Reduced heart rate variability after right-sided stroke. Stroke 1996;27:247-51.

21. Robinson TG, James $M$, Youde J, Panerai $R$, Potter J. Cardiac baroreceptor sensitivity is impaired after acute stroke. Stroke 1997;28:1671-6.

22. Devinsky O, D'Esposito M. Neurology of Cognitive and Behavioral Disorders. New York: Oxford University Press, 2004;336-351.

23. Benarroch EE. The central autonomic network: functional organization, dysfunction, and perspective. Mayo Clin Proc 1993;68:988-1001.

24. Chandra PS, Vaghania G, Bal CS, et al. Role of concordance between ictal-subtracted SPECT and PET in predicting long-term outcomes after epilepsy surgery. Epilepsy Res 2014;108:1782-9.

25. Ewing DJ, Clarke BF. Diagnosis and management of diabetic autonomic neuropathy. Br Med J (Clin Res Ed) 1982;285:916-8.

26. Deepak KK, Godbole SA, Kochhar KP, Shah P, Kochupillai N. Autonomic dysfunction and peripheral vasodilatory response in diabetes. Indian J Physiol Pharmacol 1996;40:325-9.

27. Engel J Jr. Surgical Treatment of the Epilepsies, 2nd ed. New York: Raven Press, 1993;609-621.

28. Oribe $E$, Appenzeller O. Evaluation of autonomic reflexes. In: Mohr JP, Gautier JG, eds. Guide to Clinical Neurology. New York: Churchill Livingstone, 1995;213-218.

29. Freeman R. Assessment of cardiovascular autonomic function. Clin Neurophysiol 2006;117:716-30.

30. Tinuper $P$, Bisulli $F$, Cerullo $A$, et al. Ictal bradycardia in partial epileptic seizures: autonomic investigation in three cases and literature review. Brain 2001;124(Pt 12):2361-71.

31. Rossetti AO, Dworetzky BA, Madsen JR, Golub O, Beckman JA, Bromfield EB. Ictal asystole with convulsive syncope mimicking secondary generalisation: a depth electrode study. I Neurol Neurosurg Psychiatry 2005;76:885-7.

32. Ewing DJ. Cardiovascular reflexes and autonomic neuropathy. Clin Sci Mol Med 1978;55:321-7.

33. Borst C, Wieling W, van Brederode JF, Hond A, de Rijk LG, Dunning AJ. Mechanisms of initial heart rate response to postural change. $A m$ J Physiol 1982;243:H676-81.

34. Hines EA, Brown GE. The cold pressor test for measuring the reactibility of the blood pressure data concerning 571 normal and hypertensive subjects. Am Heart J 1936;11:1-9.

35. Ghchime R, Benjelloun $H$, Kiai $H$, Belaidi $H$, Lahjouji F, Ouazzani R. Cerebral hemispheric lateralization associated with hippocampal sclerosis may affect interictal cardiovascular autonomic functions in tem- 
poral lobe epilepsy. Epilepsy Res Treat 2016;2016:7417540.

36. Hilz MJ, Devinsky O, Doyle W, Mauerer A, Dütsch M. Decrease of sympathetic cardiovascular modulation after temporal lobe epilepsy surgery. Brain 2002;125(Pt 5):985-95.

37. Dericioglu N, Demirci M, Cataltepe O, Akalan N, Saygi S. Heart rate variability remains reduced and sympathetic tone elevated after temporal lobe epilepsy surgery. Seizure 2013;22:713-8.

38. Persson $H$, Kumlien $E$, Ericson $M$, Tomson T. Circadian variation in heart-rate variability in localization-related epilepsy. Epilepsia 2007;48:917-22.

39. Persson $H$, Kumlien E, Ericson M, Tomson T. No apparent effect of surgery for temporal lobe epilepsy on heart rate variability. Epilepsy Res 2006;70:127-32.

40. Hilz MJ, Platsch G, Druschky K, et al. Outcome of epilepsy surgery correlates with sympathetic modulation and neuroimaging of the heart. J Neurol Sci 2003;216:153-62.

41. Yale SH. Antonio Maria Valsalva (1666-1723). Clin Med Res 2005;3:35-8.

42. Hallioglu O, Okuyaz C, Mert E, Makharoblidze K. Effects of antiepileptic drug therapy on heart rate variability in children with epilepsy. Epilepsy Res 2008;79:49-54.

43. Joo EY, Hong SB, Han $\mathrm{HJ}$, et al. Postoperative alteration of cerebral glucose metabolism in mesial temporal lobe epilepsy. Brain 2005;128 (Pt 8):1802-10.

44. Takaya S, Mikuni N, Mitsueda T, et al. Improved cerebral function in mesial temporal lobe epilepsy after subtemporal amygdalohippocampectomy. Brain 2009;132:185-94. 